\title{
Acceptance of AI for delegating emotional intelligence: Results from an experiment
}

\author{
Michael Leyer \\ University of Rostock \\ Queensland University of \\ Technology \\ michael.leyer@uni-rostock.de
}

\author{
Banu Aysolmaz \\ Eindhoven University of \\ Technology \\ b.e.aysolmaz@tue.nl
}

\author{
Deniz Iren \\ CAROU \\ Open Universiteit Heerlen \\ deniz.iren@ou.nl
}

\begin{abstract}
Detecting emotions of other humans is challenging for us humans. It is however important in many social contexts so that many individuals seek help in this regard. As technology is evolving, more and more AIbased options emerge that promise to detect human emotions and support decision making. We focus on the full delegation of detecting emotions to AI to contribute to our understanding how such $A I$ is perceived and why it is accepted. For this, we conduct an online scenariobased experiment in which participants have the choice to delegate emotion detection to another human in one group and to an AI tool in the other group. Our results show that the delegation rates are higher for a human, but surprisingly high for AI. The results provide insights that should be considered when designing AI-based emotion-detection tools to build trustworthy and accepted designs.
\end{abstract}

\section{Introduction}

Detecting and acting on emotions has been seen as an essential human trait [1] that specifically separates us from machines. Human decision-making is often driven by emotions of others that we perceive in a social context [2]. However, recent studies show that detecting emotions reliably is not well understood for humans and many individuals struggle with it [3]. There is no agreed-upon and standard way of identifying the emotions that manifest in a social communication setting among humans [3]. In other words, the decisionmaking affected by emotions is also subjective and volatile for humans. Hence humans may wish to delegate the emotion-detection task in various situations; e.g., under too much work strain, for cognitively complex tasks, or to avoid accountability [4$6]$.
With the recent advances in the field of Artificial Intelligence (AI), the separation of human traits from machines has become narrower [7]. AI-based emotiondetection is seen to be life-changing for our future [8]. Successful AI-based emotion-detection tools have already been designed in various domains such as healthcare [9], disaster management [10], customer service [2], social media, e-commerce, chatbots [11, $12]$, and workplace [8, 13]. However, it is rather understudied how AI-based emotion-detection is perceived and accepted by humans. Specifically, there is a lack of research for decision delegation to AI rather than decision support, when AI executes the decision autonomously based on the emotion analysis. Decision delegation differs fundamentally from support as the information is not provided to make an own informed understanding, but a person delegating is giving up his/her decision authority with all related consequences [4].

Understanding how humans perceive the delegation of emotion-detection is important to present proper AI functionality to users and build trust between users and AI [14]. Analysis of the human perception with regard to such an AI-based software is also valuable because the programming of emotion detection for AI-based software systems is based on the understanding of human emotion-detection [3]. The subjective and context-dependent nature of interpreting human emotions make it a challenging task to equip an AIbased software system with capabilities and characteristics that are trusted by its users [15]. The investigation of human perception for emotiondetection delegation tasks can help to overcome this challenge. Therefore, in this study, we investigate the research questions how humans perceive and accept the delegation of an emotion-detection task to an AI-based software and how this differs from when the task is delegated to another human, which is the typical case due to the humanly nature of the task. 
The remainder of this paper is as follows. In section 2 , we describe the theoretical background of our study. In section 3, we provide the methodological details of this research. Section 4 covers the results. In section 5 , we discuss the results. Finally, in section 6, we conclude the study and discuss the theoretical and practical implications.

\section{Theoretical background}

\subsection{Emotion detection}

Emotions play an important role in how humans think and act. They manifest in both verbal and nonverbal communication in social interactions. The abilities to perceive and convey emotions have long been thought as the essence of human nature [1] and one of the essential traits that separates humans from computers. However, the recent developments in AI have paved the way to promising research that aims at empowering software agents and robots with emotion recognition capabilities as well.

Researchers reported accuracy levels in emotion recognition on textual content that outperform human capabilities [16]. Computer vision models using deep convolutional neural networks were shown to be effective in detecting non-verbal emotional cues on facial expressions of humans [17] and on body gestures [18]. Similarly, convolutional neural networks with long-short-term memory achieved higher accuracy in detecting emotions by extracting paralinguistic information from human speech. Moreover, the detection of emotional-valence was shown to be possible by analyzing even simpler interaction methods such as keyboard strokes [19] and mouse movements [20]. Studies also show additional benefits of using a multimodal approach, i.e. using multiple forms of inputs together, such as speech, text, and video for emotion recognition [21].

It is foreseen that computers will soon be equipped with holistic capabilities of detecting human emotions and adjusting their behaviors based on the emotions of the humans that they interact with [8]. However, despite the large body of literature on automated emotion recognition, there is a lack of research on how automated emotion recognition will be perceived and accepted by humans.

\subsection{Attachment theory}

Attachment theory provides a lens to explain how humans bond to and form relations with others [22]. In line with Leyer and Schneider [23], we adopt attachment theory to explain the relation of individuals to human and AI-based software. Attachment theory studies the tendency of a person to form, maintain and dissolve loving bonds with particular other people [22, 24] and describes characteristics of a person's relationship with other people [25-27]. The theory contains that individuals are constantly looking for other people who support their needs best and ensure security. Therefore, attachment develops more likely to familiar individuals that are expected to ensure security even if the particular person is not known much [27].

Since its origins in child development and interpersonal relationships, attachment theory has been applied in other contexts. Research in psychology and marketing suggests that attachment can go beyond the context of person-to-person relationships and can extend to ownership [28], brands [29] and places [30]. As attachment builds up over time, studies have also shown that people generalize the attachment or transfer it from one context to another [31] when they evaluate a new relationship. Accordingly, studies have considered the attachment of humans to software systems to explain their attitude for assessing the system's outcome [23] and collaborating with the system [32]. Beyond companies naming their software, e.g. Alexa, humans tend to give names to objects, develop attachment to systems [33] and some could even develop a feeling of love if software is embedded in an object [34]. Hence, similar to humans, places or objects, systems are perceived as artefacts to which attachment can be built especially when they have some sort of behavior that is perceived as intelligent.

\subsection{Related work}

Decision delegation differs in relation to decision support systems insofar that the delegate executes the resulting decision of an analysis autonomously [35]. Such decision-making is likely to be delegated when an individual feels overstrained with the task due to high workload [4] or cognitive reasons [5]. The more the delegate is perceived as capable in performing the task, the higher the delegation rates become [36]. When decisions are emotional, individuals have been found to delegate to other professional humans to avoid potential guilt of own decision making [6, 37]. Humans are found to approach the decision-making delegation to computers differently than humans. There is a tendency of humans to expect perfect results from an AI-based system [38]. There is, however, also evidence that even objectively capable AI-based system are more avoided than their human counterparts [39].

To date, studies have mostly focused on the design of emotion-aware systems (e.g., for healthcare and disaster management $[9,10])$ and acknowledged the utility of delegating emotion-detection tasks to a system 
(e.g., social media conversations, chatbots, ecommerce, and shopping $[11,12,40])$. The efficacy and reliability of an emotion-detection app has been investigated with the motivation that its users will not trust the app if it does not work properly [41].

Human-computer interaction research has looked into affective reactions of people to social robots and anthropomorphized agents and the emotional response expected from these agents for an effective interaction $[42,43]$. Not much research can be found in the context of delegation of emotion detection to an AI-based system. Madsen and Gregor [44] used personal attachment as the affective component of the trust to computer scale they developed. Leyer and Schneider [23] showed that attachment theory adds to the explanation why negative decision outcomes of an AIbased system are perceived less emotional (positive and negative) compared to humans. You and Lionel [32] showed that teams perform better when they have higher emotional attachment to the robots that they work with. Henkel, et al. [2] empowered service employees with an emotion-recognition assistant for analyzing customers' emotions in real-time on the phone. This allowed the service agents to delegate the task of observing emotional cues of the customer to the emotionrecognition assistant and focus on their main tasks. In practice, emotion-detection is being provided as a service (e.g., Microsoft Azure and Affectiva.com) to be integrated in systems for seamless support and delegation of actions based on emotions [3].

Emotional distress in daily life and in the workplace increases constantly in the current era [45]. Emotional intelligence of employees in the workplace, which is the capability of understanding and managing emotions of others [46], is found to have significant importance to reduce emotional distress and improve work performance [47]. Emotional intelligence competencies have been shown to contribute to work performance even more than intellectual intelligence (36\% vs 27\%) [48]. Nevertheless, it is a challenging task for employees to track and gauge the emotions of both others [45] and themselves [8]. In a large study of two thousand employees, two thirds of the managers reported to be uncomfortable with communicating with other employees [49], which is necessary to detect their emotional status. Based on the need for the delegation of emotion-detection, new AI tools have been developed to enable people understand and respond to the emotions of others in the workplace $[8,13]$. However, despite the increasing applications of emotion-aware systems and particularly emotion detection, the perception of individuals about the delegation of emotion-detection tasks to an AI-based system has not yet been investigated.

\subsection{Hypotheses and research model}

According to the attachment theory, a new person becoming present for an individual is evaluated with regard to prior experiences. The closer the person is perceived with regard to positively experienced contexts, the higher is the attachment. This holds true even if there is no personal relationship with this specific person. If a person offers help in the form of a delegate, then this evaluation takes place. The higher the attachment with this delegate due to prior similar experiences, the more likely is a delegation. We hypothesize that when a delegate, a human or a system that takes over a decision, offers help, the individual decides to accept/reject the help based on the attachment s/he recognizes towards the delegate. Hence, we formulate $\mathrm{H} 1$ as follows:

H1: The higher the attachment with the delegate, the higher is the acceptance of the offer to use the delegate.

When it comes to artificial beings, humans tend to ascribe to robots "human-like" characteristics such as emotional states and personality [50]. It has been shown that in technological contexts, "human-like" robot behavior tends to lead to a higher degree of attachment, even if the robots are not particularly responsive [51]. Studies have also shown that adults who interact with a more human-like robot perceive it as more empathetic and trustworthy compared to a text-based virtual assistant even when it performs identically [52]. This highlights the importance of human features and attachment that also applies for AI.

While the attachment theory acknowledges that there are individual differences in attachment patterns, it suggests that the expected similarity with people manifests in attachment and makes people prefer other people than computers for positive actions [14, 15, 53]. Due to prior similar experiences of an individual to a human, we posit that the attachment of an individual is higher to another human than AI that takes over the decision based on emotion-detection. Such an attachment is rooted in prior experiences which is less likely to have happened with AI so far. Hence, for a decision-delegation task, we formulate $\mathrm{H} 2$ as follows:

H2: The attachment of an individual is higher with another human compared to an AI-based system.

As discussed, attachment has an important role in the acceptance of an offer. Since individuals and AI-based systems are assumed to be associated with different attachments [23], an acceptance or rejection is not evident because an offer is made by a human or an AIbased system. It is rather that both forms of delegates 
lead to different levels of attachment, which then has an influence on the acceptance of the offer. Hence, we formulate $\mathrm{H} 3$ as follows:

H3: Attachment mediates the likelihood of accepting an offer from a human or an AI-based system.

Figure 1 summarizes the hypotheses in our research model.

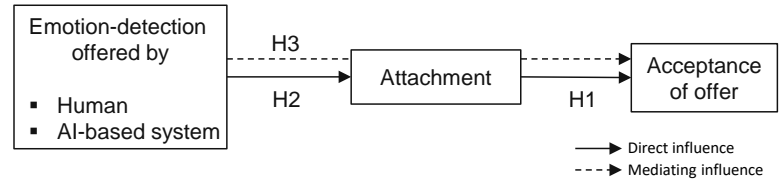

Figure 1: Research model

\section{Procedure \& method}

\subsection{Setting}

Participants were asked to accept or reject the help of a colleague/AI-based system for detecting emotions of a management board and adjusting the slides based on the detected emotions during an important presentation at work. We used a scenario as described in Appendix A-1. The participant was introduced as an employee in a small consultancy company that presents their project to the management board of a big company to win them as a client. The importance of this presentation and the gains of winning client were emphasized. The hypothetical AI-based system in this scenario is a software agent that is designed to detect the emotions of individuals based on their facial expressions and gestures while listening to the presentation. Such systems have been introduced and demonstrated in many studies (e.g., [2, 18]).

\subsection{Measures}

Participants could decide whether they would delegate the emotion detection of a management board and adjustment of the slides according to the assessment of the emotion-detection during the important presentation they make for their company. One group had the option to delegate to a colleague, the other group to an AI-based system in the form of a box. After receiving the scenario, participants were questioned regarding their level of fear (1-5) in the given situation using the scale of Izard, et al. [54].

In the following, we measured the attachment of the participant with the colleague or the AI-based system (1-5) adopting the scale of Mugge, et al. [55].
Finally, we recorded the decision made by each participant with a yes/no question whether the participant would accept or reject the offer.

Next to the control variable scared, we also measured age and gender as well as whether a participant feels intruded by the offer (single item, 1-5). As an optional open-ended comment item, we asked the participants to explain their rationale for their decision.

\subsection{Participants}

To gather participants, we used the crowd working platform Clickworker (similar to Amazon MTurk). As Clickworker is an unsupervised online platform on which participants are paid for participating in a survey, we followed the recommendations of Goodman, et al. [56] by having a short survey and including attention checks. Participants were assigned randomly to having the option to delegate to a colleague or the AI-based system.

We addressed 200 participants from Clickworker. One participant had to be deleted because of partially missing data due to technical reasons. Hence, our sample consists of 199 participants of which 34.2 per cent are male and $65.3 \%$ are female. The participants are 35.09 years old on average (SD: 10.61 ) with a minimum age of 18 and a maximum of 70. 104 participants are in the group of having the option to delegate to a colleague while 95 participants are provided with the option to delegate to the AI-based system.

\subsection{Methods}

We applied a linear regression-based mediation model analysis on our quantitative data according to our hypotheses using the Process Plugin for SPSS [57] with 5,000 samples for the bootstrapping procedure. The plugin is regression based and uses binary logistic regression for estimating the effect on the dependent variable. We coded the quantitative data in the openended comment questions. One of the authors coded all comments and identified 8 categories ( 7 themes and a none category). The two other authors categorized half of the comments each and did not identify any other themes. Related to the inter-rater reliability, a Cohen's Kappa value of 0.67 was reached, which points out to a substantial or good agreement level [58]. When two authors assigned different themes to a comment, the third author also categorized the comment. Majority decision was used for the final theme assignment.

\section{Results}

\subsection{Descriptives}


Of the 104 participants having the option to delegate to a colleague, $68.3 \%$ decided to delegate while $63.2 \%$ of the 95 participants delegated to the AI-based system. Hence, the rate is $7.5 \%$ higher for the delegation to colleagues than to the AI-based system.

The attachment with the colleague is 3.47 (SD: 0.71) which is $25.1 \%$ higher compared to the AI-based software (Mean: 2.60; SD: 0.80).

Regarding the control variables, average perceived fear in the given scenario is moderately high with 3.47 (SD: 0.75). Intrusiveness is 2.02 on average (SD: 1.17) for the colleague and 2.91 (SD: 1.22) for the AI-based system.

Table 1 provides an overview on the direct correlations between our three variables.

\begin{tabular}{|l|l|l|l|}
\hline & $(1)$ & $(2)$ & $(3)$ \\
\hline $\begin{array}{l}\text { (1) Human vs. AI-based } \\
\text { system }\end{array}$ & - & $-.50^{* * *}$ & .05 \\
\hline (2) Attachment & & - & $-0.38^{* * *}$ \\
\hline (3) Acceptance of offer & & & - \\
\hline
\end{tabular}

Table 1: Pearson correlations among the variables in the research model

\subsection{Quantitative results}

The quantitative results are calculated according to the research model and are depicted in Figure 2.

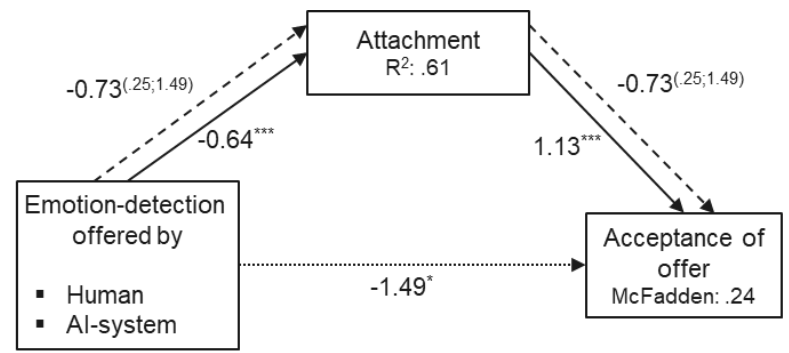

Figure 2: Results regarding the research model

The results provide empirical support for $\mathrm{H} 1$, meaning that a higher attachment with the colleague/AIbased system leads to a higher acceptance of the offer. The value of 1.13 indicates a positive influence of attachment and the McFadden value of .24 (representing the $\mathrm{R}^{2}$ for logistic regression) shows a relevant explanatory degree of our model for the acceptance of the offer.

There is also empirical support for $\mathrm{H} 2$, stating that it is more likely that there is a higher attachment with the colleague than with the AI-based system. The negative value of -0.64 shows a clear tendency of attachment in favor of humans (coded with 1 while the AI-based system is coded with 2 ) and the $\mathrm{R}^{2}$ of .61 shows a high explanation of attachment by this factor.

H3 is also supported, i.e. attachment mediates the relationship between the delegate offered and the decision to delegate. Since the value is negative with 0.73, it follows the explained logic: Humans lead to a higher attachment which then leads to a higher acceptance of the offer.

We also test the influence of the control variables outlined and find no influence of age, gender and fear on attachment or the decision. Intrusiveness is however a relevant factor, leading to a lower attachment (-.25, $\mathrm{p}$ $<.001)$ as well as lower acceptance of the offer $(-.72$, $p$ $<.001)$. This result is a first quantitative hint for the relatively more negative connotation of AI-based system compared to human colleagues.

\subsection{Qualitative results}

We identified 7 themes for the open-ended comments. 19 of the comments did not have a specific theme. Two themes were assigned for 14 comments. The themes and the number of comments related to them are shown in Table 2.

\begin{tabular}{|l|l|l|l|}
\hline Theme & All & Human & $\begin{array}{l}\text { AI- } \\
\text { based } \\
\text { System }\end{array}$ \\
\hline (1) Support as help & 81 & 43 & 38 \\
\hline (2) Support as capability & 35 & 18 & 17 \\
\hline (3) Own capability & 25 & 15 & 10 \\
\hline (4) Trust/distrust & 16 & 6 & 10 \\
\hline (5) Intrusiveness & 13 & 6 & 7 \\
\hline (6) Lack of capability & 12 & 0 & 12 \\
\hline (7) Accountability & 11 & 8 & 3 \\
\hline
\end{tabular}

Table 2: Themes and related number of comments

A high ratio of comments (42\%) cover Theme (1), pointing out that the participants appreciate the help provided by the human/AI-based system in general, the human (colleague in the scenario) being slightly more pronounced. Next, Theme (2) is covered by $18 \%$ and equally distributed among the human and the AI-based system. Thus, these participants think that the colleague/AI supports through their superior capability, which is similarly applicable for both the colleague and the AI-based system. In the next theme (3), which is observed in $13 \%$ of the comments, the participants think that they have superior capability themselves. This is valid more for a colleague rather than AI-based system. In theme (4), $8 \%$ feel trust or distrust to the colleague/AI-based system, which is applicable more to the AI. Theme (5), feeling of intrusiveness and lack of control, emerges in $7 \%$ of the comments, followed by 
Theme (6), lack of colleague/AI's capability, and Theme (7), accountability about sharing responsibility. Lack of capability theme only emerges for the AI-based system, whereas accountability is rather mentioned by the participants with a colleague's support for the emotion detection task.

\section{Discussion}

The quantitative results of our study justify that attachment felt by an individual indeed has an impact on the decision to delegate a task, whether it is another human or an AI-based system that the task is delegated to. As we expected, humans show significantly more attachment to another human rather than a system. Nevertheless, interestingly, the decision for delegation to the human and the AI-based system was different only $7.5 \%$, while overall, $66 \%$ of the participants accepted the offer for the delegation. This result may point out that individuals are getting more accustomed to incorporating AI-based systems into their lives, even for emotion-detection, which is attributed mainly to humans. It seems like the feeling of attachment is more important to accept the offer for this task rather than the fact that it is coming from a human or a system. We see that the qualitative results align with this. The fact that help is provided in this task was mostly appreciated by the participants, which seems to be much more important than assisting them with capability. This was similarly important for both delegating the task to a human and the system. Still, the provided superior capability was also valued highly and equally for both the humans and the AI-based system.

Intrusiveness, however, seems to be a prominent factor to affect both attachment and the decision to delegate, significantly more so for the AI-based system. Reactance theory may explain the sense of intrusiveness. Understanding the psychological response to conscious persuasive messaging has long been a topic of academic research [59]. However, we do not find much evidence in this regard. The qualitative results indicate that individuals might get used to the AIbased system when the situation requires them to accept the support. The functional aspects are more important than personal aspects, which explains the relatively high acceptance rate. The capability support provided by another human or an AI-based system was equally valued. Contrary to the qualitative results, intrusiveness was not a prominent theme in the comments, and it was equally observed for the human and the system.

Contrary to prior research [38], we do not find any argument for the expectation of perfection for an AIbased system but rather that individuals see a lack of capability for the AI-based system with regard to emotion detection but not at all for humans. This can be seen from an attachment perspective since participants can imagine humans from other experiences better than a never used AI-based system. Similarly, the theme of having more capability himself/herself may have emerged more for delegating the task to a human, since the individual can compare self better with a human than a system. Accountability, or feeling that the responsibility in the task is shared, can also be an important factor underlying attachment, which was notably higher for a human [6].

Our results are contrary to Yukl and Fu [36] as the perceived capability did not emerge as a main argument for delegation in the qualitative results. Our findings rather align with the argument of Schneider and Leyer [5] that participants feel overstrained in this stressful situation, shown by the high appreciation of support as help. We also do not find support for the previous results about avoidance of AI-based systems even when they are objectively capable [39], since lack of capability was only attributed to the AI-based system in our results but still the system was highly accepted by the participants. Nevertheless, we observe different underlying rationale for the delegation of the task to humans and AI-based systems, as suggested by previous studies [38, 39].

\subsection{Theoretical implications}

Our study highlights the importance of delegating emotion detection to an AI-based system despite being broadly perceived as a core human capability. We are first in addressing this aspect and find that, despite a lower attachment, individuals perceive also AI for emotion detection as broadly helpful. This may add a new perspective to the literature. We establish attachment as a new dimension in algorithmic acceptance. Based on the importance of attachment that we reveal in this study, researchers may look into ways to strengthen attachment to an AI-based system to increase its use, which can be about the time of the relation or other aspects in human-AI interaction. Our results on attachment may contribute to the adoption studies for social robots and anthropomorphized agents as well. These studies may look for ways to increase the attachment to the robots and agents relevant to their context based on the factors we have identified.

On the contrary to the studies indicating algorithmic aversion [39], our results demonstrate that individuals may actually accept an AI-based system when they need its support and develop an attachment. This may point out to the fact that the gap between AI-based systems and humans is closing, as shown by the small but still significant difference of acceptance in our results.

Our study places attachment as a new perspective in the AI adoption literature specifically focusing on decision delegation, which is rather understudied [5]. 
We also contribute by examining the underlying perceptions towards both a human and an AI-based system in a delegation task and revealing the differences. It becomes clear that functional aspects of the support provided by the delegation are similar and most important while differences of humans to AI-based systems can be explained by a higher sense of lack of capability and accountability. Distrust to AI-based system seems to be not important for emotion detection.

Overall, the high rates of decision delegation in the experiment and the appreciation of the help provided by emotion detection support indicate that many individuals are ready to delegate emotion-detection tasks to AI-based systems. This finding justifies the efforts in AI research to improve the accuracy of emotion-detection algorithms and helps to design concrete use cases for such systems.

\subsection{Practical implications}

Our results highlight the feasibility of emotion detection as a service in practice. Despite some individuals being very self-confident in their abilities, research shows that humans are not quite reliable in doing so [3]. Hence, an adequately designed AI-based system can provide an objective, standardized way that is worth to be invested in as we see quite high acceptance rates.

Our results also point out that an important aspect in acceptance/attachment with an AI-based system is the perceived lack of capability, which should be targeted by providers of such systems. The consistent capabilities of the emotion-detection system in different contexts should be demonstrated to the users, which is to be contrasted with subjective perception of humans for such a task [2]. Moreover, system providers can highlight that the use of an AI-based system would overcome the issues associated with a human when delegating an important task, namely trust and accountability.

\section{Conclusion}

Emotions play an important role during decisionmaking in serious social contexts. However, the identification of emotions is highly a subjective and arduous task even for humans. Individuals may need support for emotion-detection during strained tasks. In such situations, delegation of the decision-making based on emotion-detection may be necessary to take the strain off an individual. In the last years, various AI-based solutions have been developed for emotion-detection, which is shown to have high accuracy levels. The solutions have been implemented in diverse domains such as customer service, healthcare, chatbots, and ecommerce. However, not much research can be found on the perception of emotion-detection specifically for the delegation of a task. We turn to the attachment theory to understand the feeling of attachment and intent for acceptance for individuals when they delegate the emotion-detection to a human or an AI-based system. Our findings show that attachment felt in a situation is a relevant factor in accepting help from an AI-based system compared to humans, and individuals develop more attachment to another human than a system. Nevertheless, individuals are willing to accept the support of an AI-based system less but close to a human. We qualitatively explored the facets on which attachment and acceptance with an AI-based system is based. The fact that a support is provided for the delegation of emotion-detection and taking action upon that is valued highly for both a human and an AI-based system giving the support. A perceived superior capability being provided than the individual using the support comes the next, similar for both a human and a system. We extend prior work on decision support by providing results for emotion detection delegation. Our findings underpin how AI-based systems can be highly adopted for emotion-detection delegation. We show that an AI-based system acting as an entity based on emotion detection is perceived highly positive, not too abstract and future-oriented. The study opens up opportunities for implementing such solutions in the future and understanding the factors to make these solutions more adopted.

Our study comes along with limitations as any research. First, our study only compared a human colleague and a neutral AI. Other forms of AI such as a more humanlike AI (such as Siri or even having more of a personality to be presented to the user) could be analyzed to further explore the distinct perception with regard to emotion detection. Second, our scenario is focused on a situation in which individuals perceive the necessity of using help for emotion detection. Other scenarios in which emotion-detection is rather optional can provide different insights. Third, attachment can be dependent on the length of experience which is typically longer for humans than for an AI-based system. Given that the system provides sufficient capabilities, letting the users establish a longer relation with an AI-based system can strengthen the attachment to the system, which needs to be further examined.

\section{References}

[1] S. Turkle, The second self: Computers and the human spirit. Mit Press, 2005.

[2] A. Henkel, S. Bromuri, and D. Iren, "Half human, half machine-augmenting service employees with AI for 
interpersonal emotion regulation," Journal of Service Management, vol. In press., 2019, doi: 10.1108/JOSM05-2019-0160

[3] L. F. Barrett, R. Adolphs, S. Marsella, A. M. Martinez, and S. D. Pollak, "Emotional Expressions Reconsidered: Challenges to Inferring Emotion From Human Facial Movements," Psychological Science in the Public Interest, vol. 20, no. 1, pp. 1-68, 2019/07/01 2019, doi: $10.1177 / 1529100619832930$.

[4] C. R. Leana, "Power relinquishment versus power sharing: Theoretical clarification and empirical comparison of delegation and participation," Journal of Applied Psychology, vol. 72, no. 2, pp. 228-233, 1987.

[5] S. Schneider and M. Leyer, "Me or information technology? Adoption of artificial intelligence in the delegation of personal strategic decisions," Managerial \& Decision Economics, vol. 40, no. 3, pp. 223-231, 2019.

[6] S. Botti, K. Orfali, and S. S. Iyengar, "Tragic Choices: Autonomy and Emotional Responses to Medical Decisions," Journal of Consumer Research, vol. 36, no. 3, pp. 337-352, 2009, doi: 10.1086/598969.

[7] S. Schuetz and V. Venkatesh, "Research perspectives: The rise of human machines: How cognitive computing systems challenge assumptions of user-system interaction," Journal of the Association for Information Systems, vol. 21, no. 2, pp. 460-482, 2020, doi: 10.17705/1jais.00608.

[8] E. Whelan, D. McDuff, R. Gleasure, and J. vom Brocke, "How Emotion- Sensing Technology Can Reshape the Workplace," MITSloan Manegement Review, vol. 59, no. 3, 2018.

[9] K. Lin, F. Xia, W. Wang, D. Tian, and J. Song, "System Design for Big Data Application in Emotion-Aware Healthcare," IEEE Access, vol. 4, pp. 6901-6909, 2016, doi: 10.1109/ACCESS.2016.2616643.

[10] J. Wang, S. Wang, Y. Guo, X. Hu, X. Li, and J. Cheng, "Towards Intelligent Decision Making in Emotionaware Applications," Proceedings of the 52nd Hawaii International Conference on System Sciences, vol. 6, pp. 2326-2334, 2019, doi: 10.24251/hicss.2019.281.

[11] A. Chatterjee, U. Gupta, M. K. Chinnakotla, R. Srikanth, M. Galley, and P. Agrawal, "Understanding Emotions in Text Using Deep Learning and Big Data," Computers in Human Behavior, vol. 93, pp. 309-317, 2019/04/01/ 2019, doi: https://doi.org/10.1016/j.chb.2018.12.029.

[12] A. M. Bhatti, M. Majid, S. M. Anwar, and B. Khan, "Human emotion recognition and analysis in response to audio music using brain signals," Computers in Human Behavior, vol. 65, pp. 267-275, 2016/12/01/ 2016, doi: https://doi.org/10.1016/j.chb.2016.08.029.

[13] A. V. Simpson and M. Berti, "Exploring the Potential of AI-Assisted Organizational Compassion," 2019, vol. 2019: Academy of Management Briarcliff Manor, NY 10510, 1 ed., p. 16780.

[14] A.-M. Seeger, J. Pfeiffer, and A. Heinzl, "Designing Anthropomorphic Conversational Agents. Development and Empirical Evaluation of a Design Framework," presented at the Proceedings of the International Conference on Information Systems, Atlanta, 2018.
[15] W. Wang and I. Benbasat, "Attributions of Trust in Decision Support Technologies. A Study of Recommendation Agents for E-Commerce," Journal of Management Information Systems, vol. 24, no. 4, pp. 249-273, 2008.

[16] E. Batbaatar, M. Li, and K. H. Ryu, "Semantic-emotion neural network for emotion recognition from text," IEEE Access, vol. 7, pp. 111866-111878, 2019.

[17] E. Barsoum, C. Zhang, C. C. Ferrer, and Z. Zhang, "Training deep networks for facial expression recognition with crowd-sourced label distribution," in Proceedings of the 18th ACM International Conference on Multimodal Interaction, 2016, pp. 279-283.

[18] F. Noroozi, D. Kaminska, C. Corneanu, T. Sapinski, S. Escalera, and G. Anbarjafari, "Survey on emotional body gesture recognition," IEEE transactions on affective computing, 2018.

[19] P. Khanna and M. Sasikumar, "Recognising emotions from keyboard stroke pattern," International journal of computer applications, vol. 11, no. 9, pp. 1-5, 2010.

[20] J. Estrada, J. Buhia, A. Guevarra, and M. R. Forcado, "Keyboard and Mouse: Tools in Identifying Emotions During Computer Activities," in International Conference on Big Data Technologies and Applications, 2017: Springer, pp. 115-123.

[21] S. Poria, I. Chaturvedi, E. Cambria, and A. Hussain, "Convolutional MKL based multimodal emotion recognition and sentiment analysis," in 2016 IEEE 16th international conference on data mining (ICDM), 2016: IEEE, pp. 439-448.

[22] C. Hazan and P. R. Shaver, "Attachment as an Organizational Framework for Research on Close Relationships," Psychological Inquiry, vol. 5, no. 1, pp. 1-22, 1994/01/01 1994, doi: 10.1207/s15327965pli0501_1.

[23] M. Leyer and S. Schneider, "ME, YOU or AI? Who we trust to have the last word and how we feel about it," in Proceedings of the European Conference of Information System 2019, Stockholm, 2019, p. Paper 36.

[24] K. Bartholomew and L. M. Horowitz, "Attachment styles among young adults: a test of a four-category model," (in eng), J Pers Soc Psychol, vol. 61, no. 2, pp. 226-44, Aug 1991, doi: 10.1037//0022-3514.61.2.226.

[25] J. Bowlby, Attachment and loss: Vol. 2 Separation: Anxiety and anger. New York: Basic Books, 1973.

[26] J. Bowlby, Attachment and loss: Vol. 3. Sadness and depression. New York: Basic Books, 1980.

[27] J. Bowlby, Attachment and loss: Vol. 1. Attachment 2ed. New York: Basic Books, 1982.

[28] S. Kleine and S. Baker, "An Integrative Review of Material Possession Attachment," Academy of Marketing Science Review, vol. 1, 01/01 2004.

[29] A. Japutra, Y. Ekinci, and L. Simkin, "Positive and negative behaviours resulting from brand attachment. The moderating effects of attachment styles," European Journal of Marketing, vol. 52, no. 5/6, pp. 1185-1202, 2018.

[30] D. R. Williams and J. J. Vaske, "The Measurement of Place Attachment: Validity and Generalizability of a Psychometric Approach," Forest Science, vol. 49, no. 6, pp. 830-840, 2003, doi: 10.1093/forestscience/49.6.830. 
[31] R. C. Fraley and K. E. Davis, "Attachment formation and transfer in young adults'close friendships and romantic relationships," Personal Relationships, vol. 4, no. 2, pp. 131-144, 1997, doi: 10.1111/j.14756811.1997.tb00135.x.

[32] S. You and P. R. J. Lionel, "Emotional attachment, performance, and viability in teams collaborating with embodied physical action (EPA) robots," Journal of the Association for Information Systems, vol. 19, no. 5, pp. 377-407, 2018, doi: 10.17705/1jais.00496.

[33] N. Choi, "Information systems attachment: An empirical exploration of its antecedents and its impact on community participation intention," Journal of the American Society for Information Science and Technology, vol. 64, no. 11, pp. 2354-2365, 2013, doi: 10.1002/asi.22939.

[34] H. Geoghegan and A. Hess, "Object-love at the Science Museum: cultural geographies of museum storerooms," cultural geographies, vol. 22, no. 3, pp. 445-465, 2015/07/01 2014, doi: 10.1177/1474474014539247.

[35] J. W. Burton, M.-K. Stein, and T. B. Jensen, "A systematic review of algorithm aversion in augmented decision making," Journal of Behavioral Decision Making, vol. 33, no. 2, pp. 220-239, 2020, doi: 10.1002/bdm.2155.

[36] G. Yukl and P. P. Fu, "Determinants of delegation and consultation by managers," Journal of Organizational Behavior, vol. 20, no. 2, pp. 219-232, 1999, doi: 10.1002/(sici)1099-1379(199903)20:2<219::Aidjob922>3.0.Co;2-8.

[37] K. Orfali and E. Gordon, "Autonomy gone awry: a cross-cultural study of parents' experiences in neonatal intensive care units," Theoretical Medicine and Bioethics, vol. 25, no. 4, pp. 329-365, 2004/07/01 2004, doi: 10.1007/s11017-004-3135-9.

[38] A. Prahl and L. Van Swol, "Understanding algorithm aversion: When is advice from automation discounted?," Journal of Forecasting, vol. 36, no. 6, pp. 691-702, 2017, doi: 10.1002/for.2464.

[39] B. Dietvorst, J. P. Simmons, and C. Massey, "Algorithm aversion: People erroneously avoid algorithms after seeing them err," Journal of Experimental Psychology: General, vol. 144, no. 1, p. 114, 2015.

[40] C. Martin, L. Ball, J. Archibald, and L. Carson, "Towards the improvement of self-service systems via emotional virtual agents," presented at the Proceedings of the 26th Annual BCS Interaction Specialist Group Conference on People and Computers, Birmingham, United Kingdom, 2012.

[41] J. M. Arana, F. Gordillo, J. Darias, and L. Mestas, "Analysis of the efficacy and reliability of the Moodies app for detecting emotions through speech: Does it actually work?," Computers in Human Behavior, vol. 104, p. 106156, 2020/03/01/ 2020, doi: https://doi.org/10.1016/j.chb.2019.106156.

[42] D. B. Shank, C. Graves, A. Gott, P. Gamez, and S. Rodriguez, "Feeling our way to machine minds: People's emotions when perceiving mind in artificial intelligence," Computers in Human Behavior, vol. 98, pp. 256-266, 2019/09/01/ 2019, doi: https://doi.org/10.1016/j.chb.2019.04.001.
[43] I. Seeber, L. Waizenegger, S. Seidel, S. Morana, I. Benbasat, and P. B. Lowry, "Collaborating with technology-based autonomous agents," Internet Research, vol. ahead-of-p, no. ahead-of-print, 2020, doi: 10.1108/intr-12-2019-0503.

[44] M. Madsen and S. Gregor, "Measuring HumanComputer Trust," Proceedings of Eleventh Australasian Conference on Information Systems, pp. 6-8, 2000.

[45] B. Parks. "Tech to lower workplace stress: New AI tools for stress management can help identify at-risk employees."

https://workflow.servicenow.com/employeeengagement/technology-lower-workplace-stress/ (accessed 2020).

[46] J. D. Mayer, P. Salovey, P. Salovey, and D. Sluyter, "Emotional development and emotional intelligence: Implications for educators," What is emotional intelligence, vol. 5, 1997.

[47] A. Khalili, "The role of emotional intelligence in the workplace: A literature review," International Journal of Management, vol. 29, no. 3, p. 355, 2012.

[48] V. Dulewicz and M. Higgs, "Emotional intelligence-A review and evaluation study," Journal of managerial Psychology, 2000.

[49] L. Solomon. "Two-Thirds of Managers Are Uncomfortable Communicating with Employees." Harvard Business Review. https://hbr.org/2016/03/twothirds-of-managers-are-uncomfortable-communicatingwith-employees (accessed 2020).

[50] K. M. Lee, W. Peng, S.-A. Jin, and C. Yan, "Can Robots Manifest Personality?: An Empirical Test of Personality Recognition, Social Responses, and Social Presence in Human-Robot Interaction," Journal of Communication, vol. 56, no. 4, pp. 754-772, 2006, doi: 10.1111/j.14602466.2006.00318.x.

[51] G. E. Birnbaum, M. Mizrahi, G. Hoffman, H. T. Reis, E. J. Finkel, and O. Sass, "What robots can teach us about intimacy: The reassuring effects of robot responsiveness to human disclosure," Computers in Human Behavior, vol. 63, pp. 416-423, 2016/10/01/ 2016, doi: https://doi.org/10.1016/j.chb.2016.05.064.

[52] R. Looije, M. A. Neerincx, and F. Cnossen, "Persuasive robotic assistant for health self-management of older adults: Design and evaluation of social behaviors," International Journal of Human-Computer Studies, vol. 68, no. 6, pp. 386-397, 2010/06/01/ 2010, doi: https://doi.org/10.1016/j.ijhcs.2009.08.007.

[53] A. Waytz, J. Heafner, and N. Epley, "The mind in the machine: Anthropomorphism increases trust in an autonomous vehicle," Journal of Experimental Social Psychology, vol. 52, pp. 113-117, 2014/05/01/ 2014, doi: https://doi.org/10.1016/j.jesp.2014.01.005.

[54] C. E. Izard, D. Z. Libero, P. Putnam, and O. M. Haynes, "Stability of emotion experiences and their relations to traits of personality," Journal of Personality and Social Psychology, vol. 64, no. 5, pp. 847-860, 1993.

[55] R. Mugge, H. N. J. Schifferstein, and J. P. L. Schoormans, "Product attachment and satisfaction. Understanding consumers' post-purchase behavior," Journal of Consumer Marketing, vol. 27, no. 3, pp. 271282, 2010. 
[56] J. K. Goodman, C. E. Cryder, and A. Cheema, "Data Collection in a Flat World: The Strengths and Weaknesses of Mechanical Turk Samples," Journal of Behavioral Decision Making, vol. 26, no. 3, pp. 213224, 2012.

[57] K. J. Preacher and A. F. Hayes, "Asymptotic and resampling strategies for assessing and comparing indirect effects in multiple mediator models," Behavior Research Methods, vol. 40, no. 3, pp. 879-891, August 2008.

[58] J. R. Landis and G. G. Koch, "The Measurement of Observer Agreement for Categorical Data," Biometrics, vol. 33, no. 1, pp. 159-174, 1977, doi: 10.2307/2529310.

[59] J. W. Brehm, A theory of psychological reactance (A theory of psychological reactance.). Oxford, England: Academic Press, 1966, pp. x, 135-x, 135.

\section{Appendix}

\section{A-1: Scenario description}

You are working for a small consultancy company. You have an important pitch of your project coming up with the management board of a big company you want to win as clients. It is a prestigious project for you and your company as it will secure the income stream for the next two years and potentially your job. Your company is dependent on winning this pitch so that it will also generate enough money to pay your salary. You will be rewarded with being the project leader and have the chance to generate follow-up business which might provide you the chance of becoming a managing partner. However, all depends on you now to present your ideas after a 5 weeks of preparations. You have never seen the management board of the company before which consists of seven people. In order to convince them, you have developed several strategies of argumentation what to present during the presentation. If one strategy is not convincing, you can easily switch to a different one, but you have to know when your argumentation is not convincing and you can only change once given the time constraint. You will be pretty busy presenting the complex topic so it is very difficult to observe all board members' emotional reaction at the same time.

In this situation, an AI-based software is offered to you before the meeting. It is able to detect the emotional status of all members of the management board based on their facial expressions and gestures while listening to you. You can place the detector in front of you and no one will notice. The software will then scan the data and compare with your strategies from the content of your slides. It will then switch to the respective slide in case your first strategy does not seem to convince the present board members.
In this situation, an experienced colleague is offering you support before the meeting. He will be observing the emotional status of all members of the management board based on their facial expressions and gestures while listening to you. He knows your strategies and will then switch from the computer to the respective slide in case your first strategy does not seem to convince the present board members.

Group with AI-based software: Do you accept the help from the AI-based software to have better chances of winning the pitch?

Group with colleague: Do you accept the help from the colleague to have better chances of winning the pitch? 